\title{
Ehlers-Danlos syndrome type IV is associated with a novel G984R COL3A1 mutation
}

\author{
YAO DENG, SHIJIE WEI, SHIJUN HU, JINLAN CHEN, ZHIPING TAN and YIFENG YANG \\ Department of Cardiothoracic Surgery, The Second Xiangya Hospital, Central South University, \\ Changsha, Hunan 410011, P.R. China
}

Received February 1, 2014; Accepted November 3, 2014

DOI: $10.3892 / \mathrm{mmr} .2015 .3488$

\begin{abstract}
Ehlers-Danlos syndrome type IV is an autosomal dominant connective tissue disease. Mutations in COL3A1 have been identified to underlie this disease; however, to the best of our knowledge, no COL3A1 mutations have been reported in Ehlers-Danlos syndrome type IV patients with an ascending aortic aneurysm. In order to develop further understanding of COL3A1 mutations, an Ehlers-Danlos syndrome type IV patient diagnosed with an ascending aortic aneurysm and a familial history of sudden mortality was analyzed. Genomic DNA was isolated from the peripheral blood of the patient and his family members. All coding exons of eight aneurysm-related genes (FBN1, TGFBR1, TGFBR 2, MYH11, ACTA2, SLC2A10, NOTCH1 and COL3A1) were amplified using polymerase chain reaction (PCR). The PCR products were sequenced with the ABI 3100 Genetic Analyzer, and a mutation was predicted and identified using Polyphen-2, SIFT and Mutation Taster. The novel mutation was identified as c.2950G $>$ A in COL3A1, which results in p.G984R. All three programs predicted this mutation to be deleterous to the protein function. The novel mutation identified in this study is potentially responsible for Ehlers-Danlos syndrome type IV in this patient, and expands the spectrum of COL $3 \mathrm{~A} 1$ mutations.
\end{abstract}

\section{Introduction}

Ehlers-Danlos syndrome type IV (EDS IV; OMIM\# 130050), also known as Ehlers-Danlos syndrome, vascular type, is an autosomal dominant disorder characterized by abnormal type III collagen, thin translucent skin, arterial/intestinal/uterine fragility, extensive bruising and a characteristic facial appearance (1). The prevalence of EDS is estimated to

Correspondence to: Professor Yifeng Yang, Department of Cardiothoracic Surgery, The Second Xiangya Hospital, Central South University, 139 Middle Renmin Road, Changsha, Hunan 410011, P.R. China

E-mail: yangyifengcsuxy@gmail.com

Key words: Ehlers-Danlos syndrome type IV, COL3A1, mutation range from $1 / 10,000$ to $1 / 25,000$ and EDS IV represents 5-10\% of cases (2). As a result of spontaneous vascular/intestinal rupture, the overall life expectancy of patients with EDS IV is 48 years (3). Mutations in COL3A1, the gene that encodes type III collagen, have been associated with EDS IV. By either sequencing COL3A1 from blood samples or using biochemical tests, detection of abnormal type III collagen can identify $>95 \%$ of individuals with EDS IV (4). The two methods are used to aid in the clinical diagnosis of EDS IV (4).

Vascular complications are common in patients with EDS IV, while aortic aneurysm is a rare condition. Only five mutations of COL3A1 [c.505C >T (5), c.907G>A (6), c. $2356 \mathrm{G}>\mathrm{A}(7)$, c. $2633 \mathrm{G}>\mathrm{A}(8)$ and c.1815+5G $>\mathrm{A}(9)]$ have been reported to result in aortic aneurysm, and no variants associated with ascending aortic aneurysm have previously been reported.

The aim of the current study was to identify the first COL3A1 mutation associated with ascending aortic aneurysm in an EDS IV patient. In addition, this study summarizes all the previously reported variants of COL3A1.

\section{Patients and methods}

Ethical approval and informed consent. The current study has been approved by, and conducted according to the instructions of the Ethics Committee of Central South University (Changsha, China). The patient and the other members of his family provided written informed consent for publication.

Patient presentation. A 38-year-old male was admitted to the Department of Cardiothoracic Surgery, The Second Xiangya Hosptial (Changsha, China) due to detection of an aortic aneurysm combined with aortic incompetence by echocardiography in medical examination. Physical examination revealed that the patient had no notable symptoms except for thin translucent skin (Fig. 1C) and a soft diastolic murmur of the aortic valve. There were no associated events in the patient's past medical history. The results of an ECG and a chest x-ray were normal. His father, grandfather and one of his four uncles suffered from sudden mortality (Fig. 2A) due to aortic rupture. No other family members, including his son, had any similar conditions or experiences.

Imaging examination. Computerized tomographic angiography (CTA) of the heart and thoracic aorta was performed 
in the Second Xiangya Hospital of Central South University (Changsha, China).

Molecular genetic analysis. Genomic DNA was prepared from the peripheral blood of the patient and his family members using a DNeasy Blood \& Tissue kit (Qiagen, Valencia, CA, USA) using the QIAcube automated DNA extraction system (Qiagen, Hilden, Germany). A total of $20 \mu \mathrm{l}$ QIAGEN Proteinase and $200 \mu$ sample (whole blood in phosphate-buffered saline; Wako Pure Chemical Industries, Ltd., Osaka, Japan) was added into a $1.5 \mathrm{ml}$ microcentrifuge tube. Buffer AL (200 $\mu \mathrm{l}$; in DNeasy Blood \& Tissue Kit) was then added and the sample was mixed by pulse vortexing (HYQ-2121A; Crystal Technology \& Industries, Inc, Dallas, TX, USA) for $15 \mathrm{sec}$. The sample was then incubated at $56^{\circ} \mathrm{C}$ for $10 \mathrm{~min}$ and centrifuged at $8,000 \mathrm{x}$ g for $1 \mathrm{~min}$ to remove drops from the inside of the lid. Subsequently, $200 \mu \mathrm{l}$ ethanol (96-100\%; Sigma-Aldrich, St. Louis, MO, USA) was added to the sample and it was pulse vortexed again for $15 \mathrm{sec}$ then centrifuged at $6,000 \mathrm{x} \mathrm{g}$ for $1 \mathrm{~min}$. The sample was then added to the the QIAamp Mini spin column without wetting the rim, it was centrifuged at $6000 \mathrm{x}$ g for $1 \mathrm{~min}, 500 \mu \mathrm{l}$ Buffer AW1 was added and it was centrifuged again at $6000 \mathrm{x} \mathrm{g}$ for $1 \mathrm{~min}$. A total of $500 \mu \mathrm{l}$ Buffer AW2 was then added without wetting the rim, and the sample was centrifuged twice at 20,000 x g for $3 \mathrm{~min}$. A total of $200 \mu \mathrm{l}$ Buffer AE was then added and the sample was incubated at room temperature for $1 \mathrm{~min}$, and subsequently centrifuged at $6000 \mathrm{x}$ g for $1 \mathrm{~min}$. All buffers were from the DNeasy Blood \& Tissue kit.

All coding exons of FBN1 (NM_000138.4), TGFBR1 (NM_004612.2), TGFBR 2 (NM_003242.5), MYH11 (NM_022844.2), ACTA2 (NM_001141945.1), SLC2A10 (NM_030777.3), NOTCH1 (NM_017617.3) and COL3A1 (NM_000090.3) were amplified by the polymerase chain reaction (PCR) System 9700 (Applied Biosystems Life Technologies, Foster City, CA, USA). The amplification used $25 \mu \mathrm{l}$ reaction mixture, which consisted of $0.3 \mathrm{mM}$ deoxyribonucleotide triphosphates (BioTeke Corporation, Beijing, China), 1X PCR buffer (10 mM Tris-hydrochloric acid pH 9.0, $50 \mathrm{mM}$ potassium chloride, $0.1 \%$ Triton $\mathrm{X}-100$ and $0.01 \%$ w/v gelatin; BioTeke Corporation), $2.0 \mathrm{mM}$ magnesium chloride (BioTeke Corporation), $0.5 \mu \mathrm{M}$ each primer (forward and reverse; created by Beijing Genomics Institution, Beijing, China), 1.5 U Taq polymerase and $50 \mathrm{ng}$ genomic DNA. The thermal cycling conditions were as follows: Initial denaturation at $95^{\circ} \mathrm{C}$ for $4 \mathrm{~min}$, 35 cycles of amplification consisting of denaturation at $95^{\circ} \mathrm{C}$ for $1 \mathrm{~min}$, primer annealing at $\mathrm{X}^{\circ} \mathrm{C}$ for $30 \mathrm{sec}$ and primer extension at $72^{\circ} \mathrm{C}$ for $1 \mathrm{~min}$. A final extension step was performed at $72^{\circ} \mathrm{C}$ for $7 \mathrm{~min}$. The PCR products were sequenced by the ABI 3100 Genetic Analyzer (Applied Biosystems Life Technologies). The results of sequencing were presented using Chromas software, version 2.1.1 (http://technelysium.com.au/?page_id=13). The overlap peaks frequently indicated variants and according to the base sequence around the variant, the location of the variant in the gene was identified. Continuous overlap peaks indicated frameshift variants and single overlap peak may have indicated nonsense, synonymous or missense variants. The identified mutation was searched in Pubmed (http://www.ncbi. nlm.nih.gov/pubmed/), Google scholar (http://scholar.google. com/), dbSNP (http://www.ncbi.nlm.nih.gov/SNP/) and the Exome Sequencing Project (ESP; http://evs.gs.washington.edu/
A

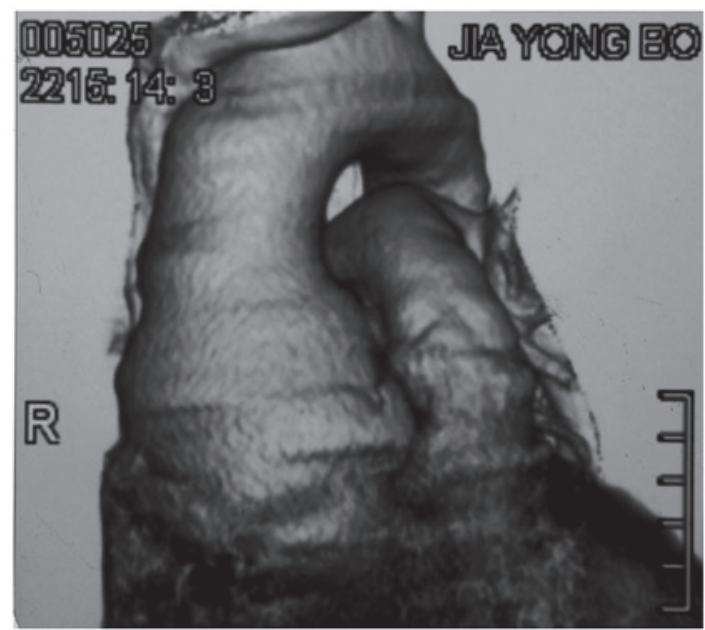

B

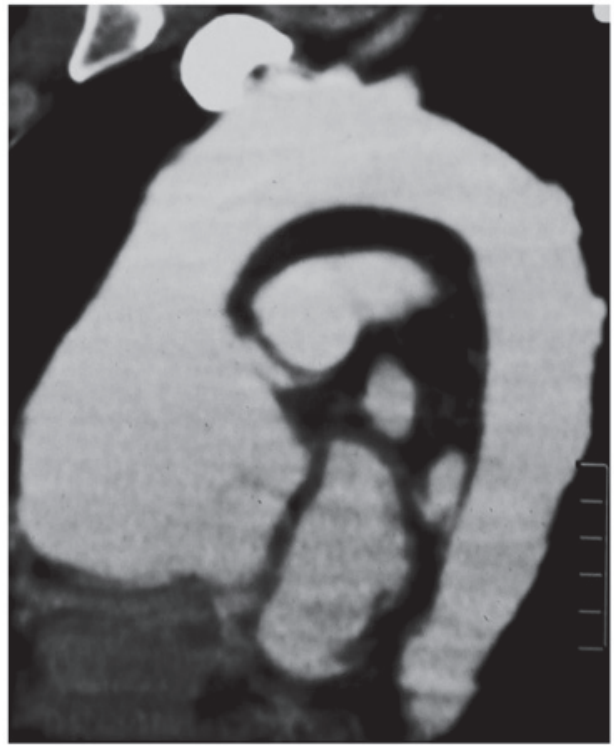

C

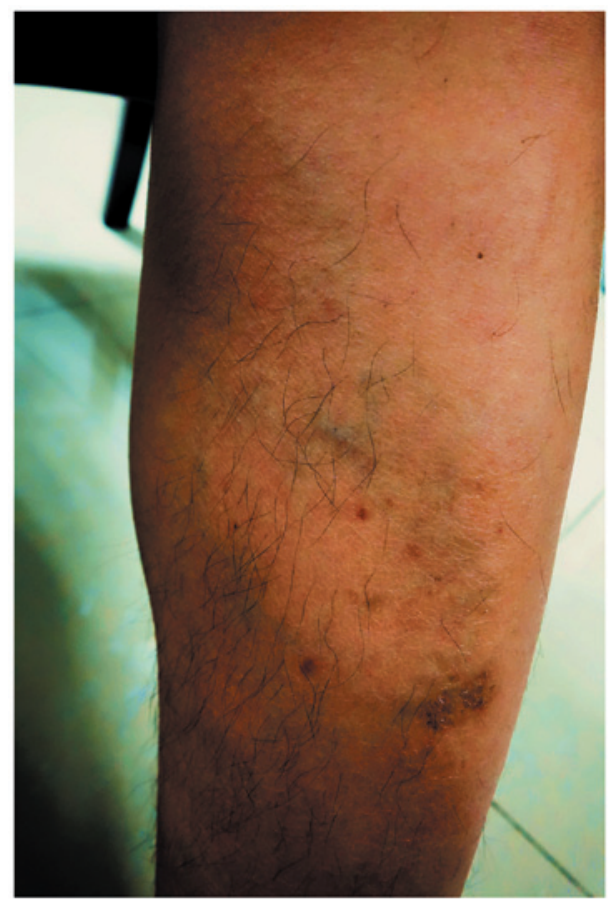

Figure 1. Two major criteria of the patient. (A) Computerised tomography (CT) reconstruction image of the ascending aorta and heart; (B) CT angiography of the ascending aorta and heart; (C) Veins visible under the thin translucent skin on the front of the left calf. 


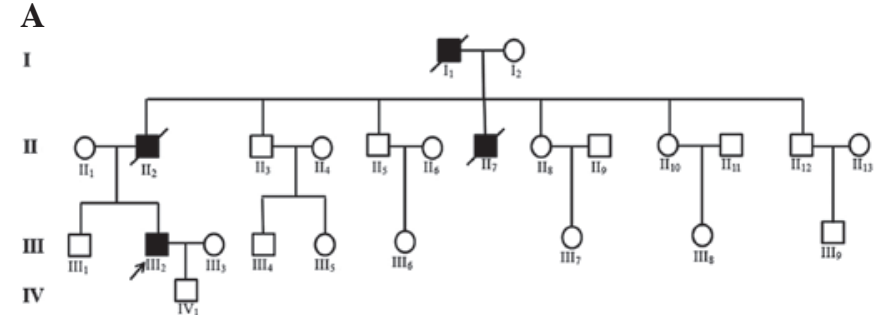

B

Control

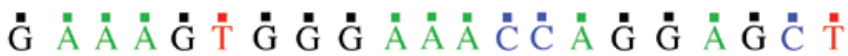

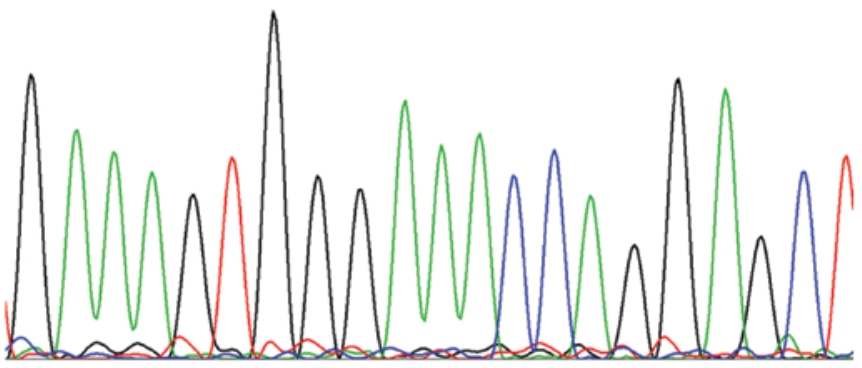

Patient

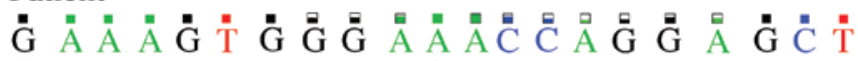

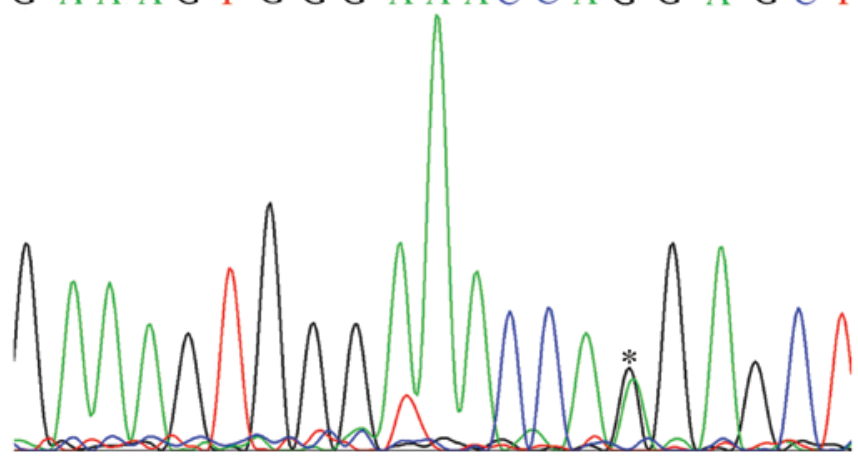

Figure 2. Patients familial history and mutation sequencing. (A) Pedigree of the family affected with EDS IV. Family members are identified by generations and numbers. Squares indicate males; circles, females; closed symbols, affected members; open symbols, unaffected members; arrow, proband; (B) Sequencing results of the COL3A1 mutation. "Sequence chromatogram indicates a $\mathrm{G}$ to A transition of nucleotide 2950.

EVS/) (10), and mutations were only considerd as candidate mutations if they had never been previously reported in humans, and were absent from dbSNP and ESP. Three online protein prediction software packages, Polyphen-2 (polymorphism phenotyping) (11), SIFT (Sorting Intolerant From Tolerant) (12) and Mutation Taster (13) were used to evaluate the effect of the novel mutations. In the current study, frameshift and nonsense variants were not observed and G984R in COL3A1 was the only candidate mutation. All of the three protein prediction software packages considered this variant to be damaging and it was located in a conserved region in COL3A1. This mutation also led to the replacement of one glycine in the (Gly-Xaa-Yaa) $\mathrm{n}$ repeat of the collagen triple helix, which was considered pathogenic in various previous studies $(3,14-21)$. Thus, G984R in COL3A1 was identified as a pathogenic mutation in the current study. The amino acid sequence of human COL3A1 from positions 977 to 996 was compared with similar regions

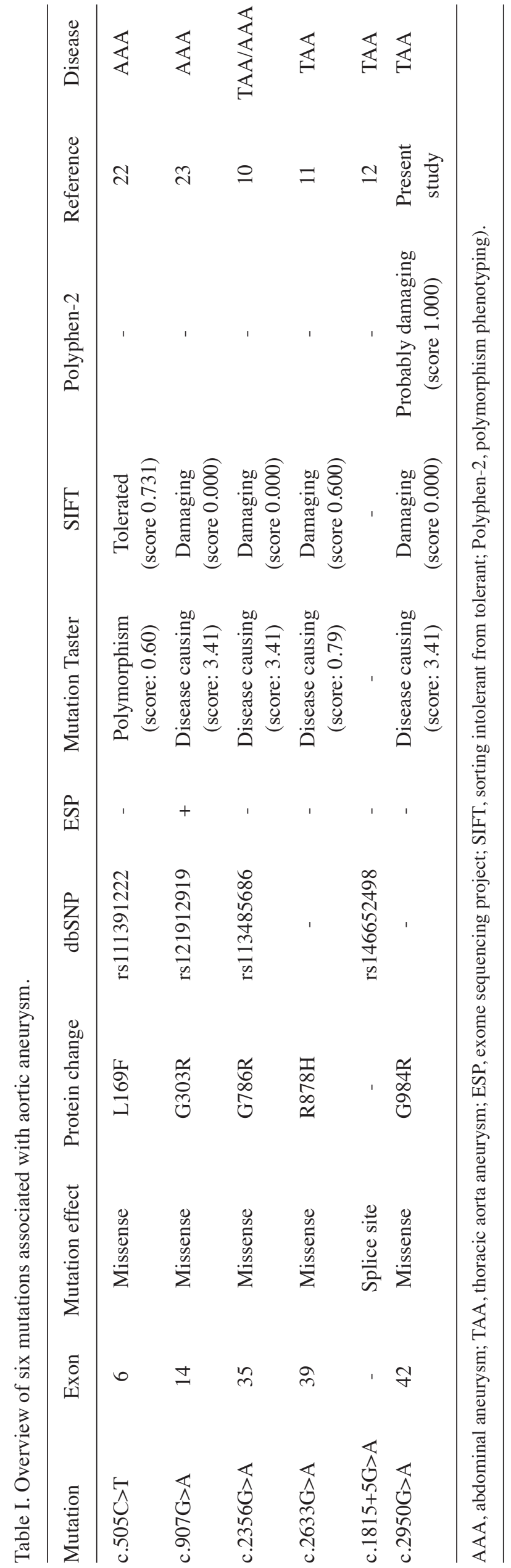


A

\begin{tabular}{|c|c|c|c|c|c|c|c|c|c|c|c|c|c|c|c|c|c|c|c|c|c|c|c|}
\hline & & & & & 977 & & & & & & & $\begin{array}{c}984 R \\
\downarrow\end{array}$ & & & & & & & & & & & \\
\hline Human & COL3A1 & . & . & . & $K$ & G & E & s & G & K & $\mathbf{P}$ & G & A & $\mathrm{N}$ & G & L & S & G & E & $\mathbf{R}$ & G & $\mathbf{P}$ & $P$ \\
\hline Chimpanzee & COL $3 A 1$ & . & . & . & K & G & E & s & G & K & $p$ & $\mathrm{~g}$ & A & N & G & L & s & G & E & $\mathbf{R}$ & G & p & $P$ \\
\hline Macaca mulatta & COL3A1 & . & . & . & K & G & E & s & G & K & p & 을 & A & N & G & L & s & G & E & $\mathbf{R}$ & $G$ & $p$ & P \\
\hline Mouse & COL3A1 & . & . & . & K & G & E & s & G & K & P & $\mathrm{G}$ & A & s & G & H & $\mathrm{N}$ & G & E & $\mathbf{R}$ & G & $\mathbf{p}$ & $P$ \\
\hline Gallus gallus & COL3A1 & . & . & . & K & G & E & D & G & K & p & $\mathrm{G}$ & v & $\mathrm{N}$ & G & v & $\mathrm{P}$ & G & E & $\mathbf{R}$ & G & A & $\mathrm{p}$ \\
\hline
\end{tabular}
- Frameshift variant
- In-frame insertion
*c. $2950 \mathrm{C}>\mathrm{A}$
- Nonsense variant
- Splice site
synonymousvariant
- Missense variant

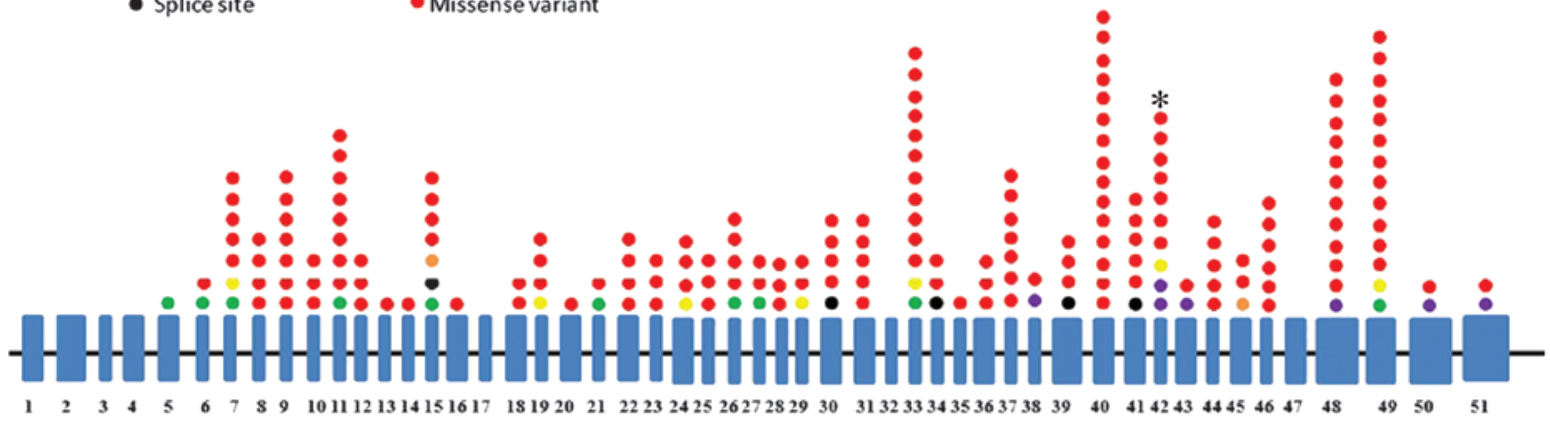

Figure 3. COLA1 protein and mutations in the COLA1 gene. (A) Alignment of multiple COL3A1 protein sequences across species. The G984 affected amino acid is located in the conserved amino acid region in different mammals (from Ensembl). The black column shows the G984 site. (B) Distribution of the 204 reported variants and the novel mutation in the exons of COL3A1. Circles with different colors represent variants with a matched mutation effect. "Novel mutation c.2950C $>\mathrm{A}$ identified in this article. Blue rectangles represent the exons of COL3A1 and the numbers under them denote the exon order.

in the chimpanzee, Macaca mulatta, mouse and Gallus gallus using Ensembl (http://asia.ensembl.org/index.html).

Variants summary. The variants previously reported in COL3A1 were searched in Pubmed in order to identify mutations associated with aortic aneurysm. All of the variants were matched in dbSNP and ESP to identify variants previously associated with disease in healthy populations. Mutations leading to aortic aneurysm were evaluated by Polyphen-2, SIFT and Mutation Taster.

\section{Results}

Result of computerized tomographic angiography (CTA). The images from the CTA revealed that the root of the ascending aorta was dilated and had formed a sacculated aortic aneurysm (Fig. 1A and B) with a diameter of $>7 \mathrm{~cm}$.

Novel mutation and functional prediction. Sequencing the PCR products of the eight aortic aneurysm-related genes, mentioned in Patients and methods, identified the nucleotide A instead of $\mathrm{G}$ at position 2950 of the coding sequence (c.2950G $>$ A, Fig. 2B) in COL3A1, leading to an amino acid change from glycine to arginine at position 984 of the protein (p.G984R). This c.2950G $>A$ mutation was only present in the patient and not in his family members or our control cohorts as well as dbSNP and ESP. A search of Pubmed and Google Scholar revealed that this mutation has not been previously reported. The prediction results of Polyphen-2, SIFT and Mutation Taster were probably damaging, damaging and disease causing, respectively (Table I). Alignment of multiple COL3A1 protein sequences across species showed the G984 affected amino acid is located in the conserved amino acid region in different mammals (Fig. 3A).

Variants review. A literature review revealed that 268 variants, including 66 variants in the introns and 204 variants in the exons
Table II. Diagnostic criteria for EDS IV caused by abnormal type III collagen.

Major criteria Minor criteria

Thin translucent skin Acrogeria

Arterial/intestinal/uterine fragility or rupture

Extensive bruising Characteristic facial appearance

$$
\text { Hypermobility of small joints }
$$
Tendon and muscle rupture Clubfoot Early-onset varicose veins Pneumothorax Gingival recession Positive family history/sudden death Arteriovenous, carotid-cavernous sinus fistula

(Fig. 3B), were identified in COL3A1. Of the 268 mutations, 257 variants were previously reported to be pathogenic. In total, $1.9 \%(5 / 257)$ of the mutations were found in the ESP. Of the exon variants, 159 of 204 variants lead to the replacement of one glycine in the (Gly-Xaa-Yaa)n repeat of the collagen triple helix. All of the 159 missense variants have been previously reported as pathogenic and were not found in the ESP database. In total, five mutations [c.505C $>$ T (5), c.907G $>$ A (6), c.2356G >A (7), c. $2633 \mathrm{G}>\mathrm{A}(8)$ and c. $1815+5 \mathrm{G}>\mathrm{A}(9)]$ were reported as causes of aortic aneurysm. The results of functional prediction by Polyphen-2, SIFT and Mutation Taster are listed in Table I.

\section{Discussion}

Due to vascular/intestinal rupture, EDS IV is the most severe type among the different types of EDS. According to the 
Villefranche nosology (1) (Table II), the combination of two major criteria is highly specific to this disease. The patient described in this study had an aortic aneurysm combined with thin translucent skin. Hence, his clinical diagnosis was EDS IV. The molecular genetic analysis revealed a mutation in COL3A1, which confirmed the diagnosis. In order to avoid aneurysmal rupture, the ascending aorta of the patient was replaced with an artificial aorta and three weeks later, following recovery, the patient was discharged from hospital.

Since Richards et al (24) initially described a variant of COL3A1 in EDS IV patients, 268 variants, including 66 intron variants and 204 exon variants (Fig. 3B), were identified in COL3A1. Of the exon variants, 159/204 variants led to the replacement of one glycine in the (Gly-Xaa-Yaa)n repeat of the collagen triple helix. All of the 159 missense variants have been previously reported as pathogenic and were not present in the ESP database. In total, five mutations [c.505C >T (5), c.907G >A (6), c.2356G >A (7), c.2633G >A (8) and c. $1815+5 \mathrm{G}>\mathrm{A}(9)]$ were reported as causes of aortic aneurysm. The results of functional prediction by Polyphen-2, SIFT and Mutation Taster are listed in Table I. However, they were all found in the ESP database. Whole exome data from the National Heart, Lung, and Blood Institute Grand Opportunity (NHLBI GO) ESP provided sequencing results of all protein-coding regions in 6,503 individuals without heart disease (12). Any reported pathogenic mutation identified in the ESP database may be false positive. This idicates that these five EDS IV-associated mutations may be SNPs or non-pathogenic.

To the best of our knowledge, the c.2950G $>$ A mutation identified in this study has not been previously reported, and is not present in the ESP database and control cohorts. This mutation led to an amino acid change from glycine to arginine at position 984 of the protein (p.G984R), which may damage the formation of the triple helix. The prediction results of the Polyphen-2, SIFT and Mutation Taster programs were that the mutation may be damage or disease causing. A review of the literature revealed that all the missense variants leading to the replacement of one glycine in the (Gly-Xaa-Yaa)n repeat of the collagen triple helix were previously reported to be pathogenic. Hence, c. $2950 \mathrm{G}>\mathrm{A}$ is considered to be the cause of EDS IV in this patient, which expands the spectrum of COL3A1 mutations and may make a contribution to the genetic counseling of EDS IV in the future.

COL3A1 encodes a procollagen molecule, proa1 (III); basic collagen synthesis requires three polypeptide procollagen chains, referred to as $\alpha$ chains, to be folded tightly into a triple helix (3). Every third amino acid in the protein triple helix is a glycine, and substitution of a glycine slows down formation of the triple helix (25). This may lead to collagen III breakdown or over modification. Collagen III is a major component of the extracellular matrix in skin, blood vessels and a variety of internal organs. Abnormal collagen III may result in thin translucent skin and thin artery walls, which leads to a number vascular complications, including aneurysm, artery dissection and so on. Vascular complications are commonly observed in EDS IV, while ascending aortic aneurysm is extremely rare. Hetzer et al (26) reported an isolated giant ascending aortic aneurysm in an infant with EDS IV. The infant underwent replacement of the ascending aorta and proximal aortic arch.
Genetic analysis was not performed. To the best of our knowledge, this is the only other report of an EDS IV patient with ascending aortic aneurysm, which means the novel mutation identified in our study is the first COL3A1 mutation reported in association with ascending aortic aneurysm.

To date, including the novel mutation identified in this study, only six mutations in COL3A1 have been found to be associated with aortic aneurysms (5-9). With the exception of c. $907 \mathrm{G}>\mathrm{A}$ identified in ESP, the mutations are distributed in different regions of COL3A1, and the correlation between aortic aneurysm and these specific mutations remains unknown. Thus, these associations require further investigation.

In conclusion, the results of this study revealed a novel COL3A1 mutation (p.G984R) in an EDS IV patient with ascending aortic aneurysm, which expands the spectrum of CO13A1 mutations and may aid with the genetic counseling and diagnosis of EDS IV in the future.

\section{Acknowledgements}

The authors would like to thank Miss Wang Jian for her assistance in collecting blood samples, and additionally would like to thank Mr Fan Liangliang and Mr Huang Hao for their assistance in the statistical analysis.

\section{References}

1. Beighton P, De Paepe A, Steinmann B, Tsipouras P and Wenstrup RJ: Ehlers-Danlos syndromes: revised nosology, Villefranche, 1997. Ehlers-Danlos National Foundation (USA) and Ehlers-Danlos Support Group (UK). Am J Med Genet 77: 31-37, 1998

2. Germain DP: Ehlers-Danlos syndrome type IV. Orphanet J Rare Dis 2: 32, 2007.

3. Pepin M, Schwarze U, Superti-Furga A and Byers PH: Clinical and genetic features of Ehlers-Danlos syndrome type IV, the vascular type. N Engl J Med 342: 673-680, 2000.

4. Pepin M and Byers P: Ehlers-Danlos syndrome type IV. Gene Reviews 2011. http://www.ncbi.nlm.nih.gov/books/NBK1494/. [Accessed November 20, 2013].

5. Anderson DW, Tromp G, Kuivaniemi H, et al: A type III procollagen gene mutation in a patient with late onset aneurysms. Matrix Biology 14: 392, 1994.

6. Tromp G, Wu Y, Prockop DJ, et al: Sequencing of cDNA from 50 unrelated patients reveals that mutations in the triple-helical domain of type III procollagen are an infrequent cause of aortic aneurysms. J Clin Invest 91: 2539-2545, 1993.

7. Kontusaari S, Tromp G, Kuivaniemi H, Romanic AM and Prockop DJ: A mutation in the gene for type III procollagen (COL3A1) in a family with aortic aneurysms. J Clin Invest 86: 1465-1473, 1990.

8. Kathiravel U, Keyser B, Hoffjan S, et al: High-density oligonucleotide-based resequencing assay for mutations causing syndromic and non-syndromic forms of thoracic aortic aneurysms and dissections. Mol Cell Probes 27: 103-108, 2013.

9. Sakai H, Suzuki S, Mizuguchi T, et al: Rapid detection of gene mutations responsible for non-syndromic aortic aneurysm and dissection using two different methods: resequencing microarray technology and next-generation sequencing. Hum Genet 131: 591-599, 2012.

10. Exome Variant Server, NHLBI GO Exome Sequencing Project (ESP), Seattle, WA (URL: http://evs.gs.washington.edu/EVS/) [Accessed November 20, 2013].

11. Sunyaev S, Ramensky V and Bork P: Towards a structural basis of human non-synonymous single nucleotide polymorphisms. Trends Genet 16: 198-200, 2000.

12. Ng PC and Henikoff S: SIFT: Predicting amino acid changes that affect protein function. Nucleic Acids Res 31: 3812-3814, 2003.

13. Schwarz JM, Rödelsperger C, Schuelke M and Seelow D: Mutation Taster evaluates disease-causing potential of sequence alterations. Nat Methods 7: 575-576, 2010. 
14. Pepin M, Schwarze U, Superti-Furga A and Byers PH: Clinical and genetic features of Ehlers-Danlos syndrome type IV, the vascular type. N Engl J Med 342: 673-680, 2000.

15. Drera B, Zoppi N, Ritelli M, et al: Diagnosis of vascular EhlersDanlos syndrome in Italy: clinical findings and novel COL3A1 mutations. J Dermatol Sci 64: 237-240, 2011

16. Eder J, Laccone F, Rohrbach M, Giunta C, Aumayr K, Reichel C and Trautinger F: A new COL3A1 mutation in Ehlers-Danlos syndrome type IV. Exp Dermatol 22: 231-234, 2013.

17. Kroes HY, Pals G and van Essen AJ: Ehlers-Danlos syndrome type IV: unusual congenital anomalies in a mother and son with a COL3A1 mutation and a normal collagen III protein profile. Clin Genet 63: 224-227, 2003.

18. Palmeri S, Mari F, Meloni I, et al: Neurological presentation of Ehlers-Danlos syndrome type IV in a family with parental mosaicism. Clin Genet 63: 510-515, 2003.

19. Sa YJ, Kim YD, Moon SW, Kim CK and Ki CS: Occlusive vascular Ehlers-Danlos syndrome accompanying a congenital cystic adenomatoid malformation of the lung: report of a case. Surg Today 43: 1467-1469, 2013.

20. Rebelo M, Ramos L, Lima J, et al: Ehlers-Danlos syndrome type IV in association with a (c.970G $>$ A) mutation in the COL3A1 gene. Acta Med Port 24: 1079-1086, 2011.
21. Kashizaki F, Hatamochi A, Kamiya K, Yoshizu A and Okamoto H: Vascular-type Ehlers-Danlos syndrome caused by a hitherto unknown genetic mutation: a case report. J Med Case Rep 7: 35, 2013.

22. Tan ZP, Huang C, Xu ZB, Yang JF and Yang YF: Novel ZFPM2/FOG2 variants in patients with double outlet right ventricle. Clin Genet 82: 466-471, 2012.

23. Chen J, Li B, Yang Y, et al: Mutations of the TGFBR2 gene in Chinese patients with Marfan-related syndrome. Clin Invest Med 33: E14-E21, 2010

24. Richards AJ, Ward PN, Narcisi P, et al: A single base mutation in the gene for type III collagen (COL3A1) converts glycine 847 to glutamic acid in a family with Ehlers-Danlos syndrome type IV: an unaffected family member is mosaic for the mutation. Hum Genet 89: 414-418, 1992.

25. Mizuno K, Boudko S, Engel J and Bächinger HP: Vascular Ehlers-Danlos Syndrome mutations in type III collagen differently stall the triple helical folding. J Biol Chem 288: 19166-19176, 2013

26. Hetzer R, Delmo Walter EM, Meyer R and Alexi-Meskishvili V: Isolated giant aortic aneurysm in an infant: Ehlers-Danlos Syndrome Type IV. Ann Thorac Surg 86: 632-634, 2008. 